\title{
Knockdown of survivin contributes to antitumor activity in cisplatin-resistant ovarian cancer cells
}

\author{
LI JIANG $^{1}$, RUO-YU LUO ${ }^{1}$, JING YANG ${ }^{2}$ and YAN-XIANG CHENG ${ }^{1}$ \\ ${ }^{1}$ Department of Obstetrics and Gynecology; ${ }^{2}$ Reproductive Medical Center, \\ Renmin Hospital of Wuhan University, Wuhan 430060, P.R. China
}

Received July 26, 2012; Accepted November 15, 2012

DOI: $10.3892 / \mathrm{mmr} .2012 .1216$

\begin{abstract}
Survivin (SVV) is an important member of the inhibitor of apoptosis family. It is overexpressed in a number of cancer types, including human ovarian carcinomas. SVV promotes invasion, metastasis, growth and survival of malignant cells and confers resistance to specific chemotherapeutic drugs. The present study aimed to elucidate the role and possible mechanisms of SVV in cisplatin-resistant ovarian cancer cells (A2780/CP). Using a loss-of-function approach, we investigated the effects of adenovirus-mediated knockdown of SVV by small hairpin RNA (ad5-SVV) on the expression of pro-caspase-3, cleaved caspase-3, proliferating cell nuclear antigen (PCNA) and matrix metalloproteinase-2 (MMP-2) in A2780/CP cells by real-time PCR and western blot analysis. Proliferation was measured by MTT assay, invasive potential by Transwell, and cell apoptosis by FITC-Annexin V and propidium iodide for the functional analysis of A2780/CP cells following infection with ad5-SVV. As a result, knockdown of SVV downregulated the expression of PCNA and MMP-2 and upregulated the expression of pro-caspase-3 and cleaved caspase-3. In addition, knockdown of SVV enhanced cisplatininduced proliferative activities, induced cell apoptosis and inhibited the invasive potential in A2780/CP cells. The present findings demonstrate that knockdown of SVV contributes to antitumor activity in cisplatin-resistant ovarian cancer cells via the downregulation of PCNA and MMP-2 expression and the upregulation of caspase-3 expression and indicate that SVV is a potential target for therapeutic anticancer drugs.
\end{abstract}

\section{Introduction}

Ovarian cancer is the fourth leading cause of cancer mortalities worldwide (1). Cisplatin and its analogs are first-line

Correspondence to: Professor Ruo-Yu Luo, Department of Obstetrics and Gynecology, Renmin Hospital of Wuhan University, Jiefang Road 238, Wuhan 430060, P.R. China

E-mail: luoruoyu027@126.com

Key words: survivin, cisplatin, ovarian cancer, apoptosis, proliferation chemotherapeutic agents for the treatment of human ovarian cancer $(2,3)$. Although the mechanisms involved in cisplatin resistance have yet to be comprehensively explored (4), decreased cell detoxication and defects in intracellular or extracellular survival/apoptotic pathways have been suggested to play a role in the development of resistance to cisplatin (5).

Survivin (SVV) is a unique member of the inhibitor of apoptosis (IAP) protein family. Abnormal IAP during homeostasis is considered a critical step in the initiation of cancer. SVV is an anticancer target due to its high expression in numerous types of cancer. SVV is expressed in ovarian cancer and correlates with its clinicopathological, surgical and apoptosis-related parameters (6). SVV mRNA expression levels correlate with the clinical stage, differentiation grade and lymph node metastasis, but not with histological type in ovarian cancer (7). Serum SVV reflects the peritoneal metastasis of serous ovarian cancer and may be useful as a prognostic biomarker (8). Nuclear SVV expression is a strong independent prognostic marker for poor clinical outcomes in epithelial ovarian carcinoma (9), however, a previous study demonstrated that nuclear SVV expression predicts improved outcome in pre-chemotherapy patients with metastatic ovarian cancer (10). In addition, among ovarian cancer patients receiving a taxol/platinum-based regimen, SVV expression correlates with a lower clinical or pathological complete remission rate, indicative of a direct link between SVV expression and tumor cell susceptibility to taxol (11). Nuclear SVV expression is a positive prognostic factor in taxane-platinumtreated ovarian cancer patients (12).

Notably, downregulation of SVV appears to be an effective gene therapy approach in the treatment of ovarian cancer (13). SVV and Granzyme B-induced apoptosis may function as potent agents for the treatment of primary and recurrent human ovarian carcinoma (14). SVV is important in resistance to chemotherapy and radiotherapy in ovarian cancer and in the progression of ovarian tumors and provides a pivotal prognostic implication for epithelial ovarian carcinomas (15). To date, evidence has confirmed the role and possible mechanisms of SVV in cisplatin-resistant ovarian cancer cells (15). In the present study, using a loss-of-function approach, we investigated the effects of adenovirus-mediated knockdown of SVV on the expression of pro-caspase-3, cleaved caspase-3, PCNA and MMP-2 in A2780/CP cells by real-time PCR and western blot analysis. Proliferation was measured by MTT 
assay, invasive potential by Transwell, and cell apoptosis by FITC-Annexin V and propidium iodide (PI) were performed for the functional analysis of A2780/CP cells following infection with ad5-SVV.

\section{Materials and methods}

Materials. The A2780/CP cell line was obtained from the Institute of Biochemistry and Cell Biology (Shanghai, China). Adenovirus-mediated SVV shRNA vector, negative control vector and virion-packaging elements were purchased from Genechem (Shanghai, China). Primers for SVV, PCNA, cleaved caspase-3 and MMP-2 were synthesized by Advanced Biotechnologies, Inc. (Columbia, MD, USA). Antibodies were from Santa Cruz Biotechnology, Inc. (Santa Cruz, CA, USA).

Drugs and reagents. Cisplatin was purchased from SigmaAldrich (St. Louis, MO, USA). Dulbecco's Modified Eagle's medium (DMEM) and fetal bovine serum (FBS) were purchased from Thermo Fisher Scientific (Waltham, MA,USA); TRIzol reagent and Lipofectamine 2000 were from Invitrogen (Carlsbad, CA, USA); M-MLV Reverse Transcriptase was from Promega Corp. (Madison, WI, USA); SYBR-Green Master Mixture was from Takara Bio, Inc. (Shiga, Japan). A cell apoptosis kit, PI, RNase A and Annexin V-FITC were obtained from KeyGEN Biology (Nanjing, China).

Cell culture and transfection. A2780/CP cells were cultured in DMEM supplemented with $10 \%$ heat-inactivated FBS, $100 \mathrm{U} /$ $\mathrm{ml}$ penicillin and $100 \mu \mathrm{g} / \mathrm{ml}$ streptomycin. Cells were placed in a humidified atmosphere containing $5 \% \mathrm{CO}_{2}$ at $37^{\circ} \mathrm{C}$. Ad5-SVV and negative control adenovirus were used to transfect A2780/ $\mathrm{CP}$ cells. Cells were subcultured at a 1:5 dilution in $300 \mu \mathrm{g} /$ ml G418-containing medium. Positive stable transfectants were selected and expanded for further analysis. The clone in which Ad5-SVV vectors were transfected was designated as Ad5-SVV, the negative control vectors transfected were designated as NC, and A2780/CP cells were designated as CON.

Quantitative real-time PCR. To quantitatively determine the mRNA expression level of SVV in A2780/CP cell lines, real-time PCR was performed. Total RNA from each clone was extracted with TRIzol according to the manufacturer's instructions. Reverse transcription was performed using M-MLV and cDNA amplification was carried out using a SYBR Green Master Mix kit according to the manufacturer's instructions. The SVV gene was amplified using specific oligonucleotide primers and the human GAPDH gene was used as an endogenous control. PCR primer sequences were as follows: SVV, 5'-ACCAGGTGAGAAGTGAGGGA-3' and 5'-AACAGTAGAGGAGCCAGGGA-3'; PCNA, 5'-CCATCC TCAAGAAGGTGTTGG-3' and 5'-GTGTCCCATATCCGC AATTTTAT-3'; cleaved caspase-3, 5'-AGAGGGGATCGTTG TAGAAG-3' and 5'-GTTGCCACCTTTCGGTTAAC-3'; MMP-2, 5'-GGCCCTGTCACTCCTGAGAT-3' and 5'-GGC ATCCAGGTTATCGGGGA-3'; GAPDH, 5'-CAACGAATT TGGCTACAGCA-3' and 5'-AGGGGTCTACATGGCAA CTG-3'. Data were analyzed using the comparative $\mathrm{Ct}$ method $\left(2^{-\Delta \Delta \mathrm{Ct}}\right)$. Three separate experiments were performed for each clone.
Western blot analysis. A2780/CP cells were harvested and extracted using lysis buffer (Tris-HCl, SDS, mercaptoethanol, glycerol). Cell extracts were boiled for $5 \mathrm{~min}$ in loading buffer and then equal amounts of cell extracts were separated by $15 \%$ SDS-PAGE. Separated protein bands were transferred onto polyvinylidene fluoride membranes and the membranes were blocked in 5\% skimmed milk powder. Primary antibodies against SVV, PCNA, pro-caspase-3, cleaved caspase-3 and MMP-2 were diluted according to the manufacturer's instructions and applied to membranes overnight at $4^{\circ} \mathrm{C}$. Following this, horseradish peroxidase-linked secondary antibodies were added at a dilution ratio of 1:1,000 and incubated at room temperature for $2 \mathrm{~h}$. Membranes were washed with PBS three times and the immunoreactive bands were visualized using ECL-PLUS kit according to the manufacturer's instructions. Relative protein levels in various cell lines were normalized to GAPDH concentration. Three separate experiments were performed for each clone.

Cell proliferation assay. Cell proliferation was analyzed with the MTT assay. Briefly, cells infected with ad5-SVV virus were incubated in 96-well plates at a density of $1 \times 10^{5}$ cells/ well with DMEM supplemented with $10 \%$ FBS. Cells were treated with $20 \mu \mathrm{l}$ MTT dye at $0,24,48,72,96$ and $120 \mathrm{~h}$ and then incubated with $150 \mu \mathrm{l}$ DMSO for $5 \mathrm{~min}$. The color reaction was measured at $570 \mathrm{~nm}$ with an enzyme immunoassay analyzer (Bio-Rad, Hercules, CA, USA). Proliferation activity was calculated for each clone.

Transwell invasion assay. Transwell filters were coated with matrigel $(3.9 \mu \mathrm{g} / \mu \mathrm{l}, 60-80 \mu \mathrm{l})$ on the upper surface of a polycarbonic membrane (diameter, $6.5 \mathrm{~mm}$; pore size, $8 \mu \mathrm{m}$ ). Following incubation at $37^{\circ} \mathrm{C}$ for $30 \mathrm{~min}$, the matrigel solidified and served as the extracellular matrix for analysis of tumor cell invasion. Harvested cells $\left(1 \times 10^{5}\right)$ in $100 \mu 1$ DMEM were added into the upper compartment of the chamber. A total of $200 \mu \mathrm{l}$ conditioned medium derived from NIH3T3 cells was used as a source of chemoattractant and was placed in the bottom compartment of the chamber. Following $24 \mathrm{~h}$ incubation at $37^{\circ} \mathrm{C}$ with $5 \% \mathrm{CO}_{2}$, the medium was removed from the upper chamber. The non-invaded cells on the upper side of the chamber were scraped off with a cotton swab. Cells that had migrated from the matrigel into the pores of the inserted filter were fixed with $100 \%$ methanol, stained with hematoxylin and mounted and dried at $80^{\circ} \mathrm{C}$ for $30 \mathrm{~min}$. The number of cells invading through the matrigel was counted in three randomly selected visual fields from the central and peripheral portion of the filter using an inverted microscope (x200 magnification). Each assay was repeated three times.

Cell apoptosis analysis. To detect cell apoptosis, A2780/CP cells treated with ad5-SVV virus were trypsinized, washed with cold PBS and resuspended in binding buffer according to the manufacturer's instructions. FITC-Annexin V and PI were added to the fixed cells for $20 \mathrm{~min}$ in the dark at room temperature. Following this, Annexin V binding buffer was added to the mixture prior to fluorescence measurement by FACsort flow cytometer. Cell apoptosis was analyzed using CellQuest software (BD Biosciences, CA, USA). Three separate experiments were performed for each clone. 
A

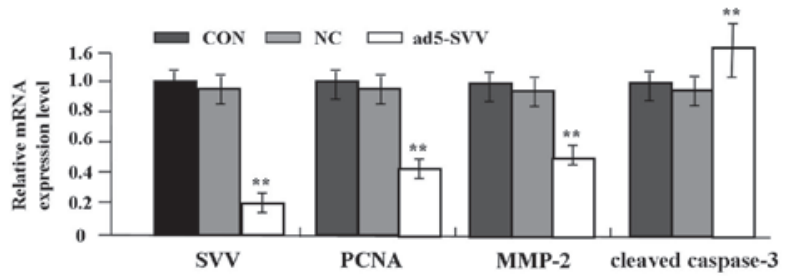

B

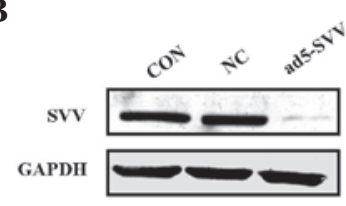

C

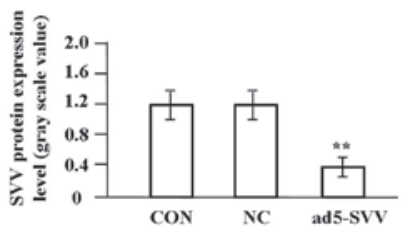

Figure 1. Effect of SVV knockdown on PCNA, cleaved caspase-3 and MMP-2 expression. (A) Expression levels of SVV, PCNA, cleaved caspase-3 and MMP-2 mRNA were measured in A2780/CP cells by real-time PCR, demonstrating that expression of SVV, PCNA and MMP-2 mRNA was decreased, while that of cleaved caspase- 3 was increased in ad5-SVV compared with NC and CON groups (each ${ }^{* *} \mathrm{P}<0.01$ ). (B and $\mathrm{C}$ ) Western blot analysis demonstrates that the expression level of SVV protein was markedly downregulated in ad5-SVV compared with that in the NC and CON groups in A2780/CP cells ( $\left.{ }^{* *} \mathrm{P}<0.01\right)$. CON, A2780/CP cells; NC, transfected negative control vectors; Ad5-SVV, transfected Ad5-SVV vectors. SVV, survivin; PCNA, proliferating cell nuclear antigen; MMP-2 matrix metalloproteinase-2.
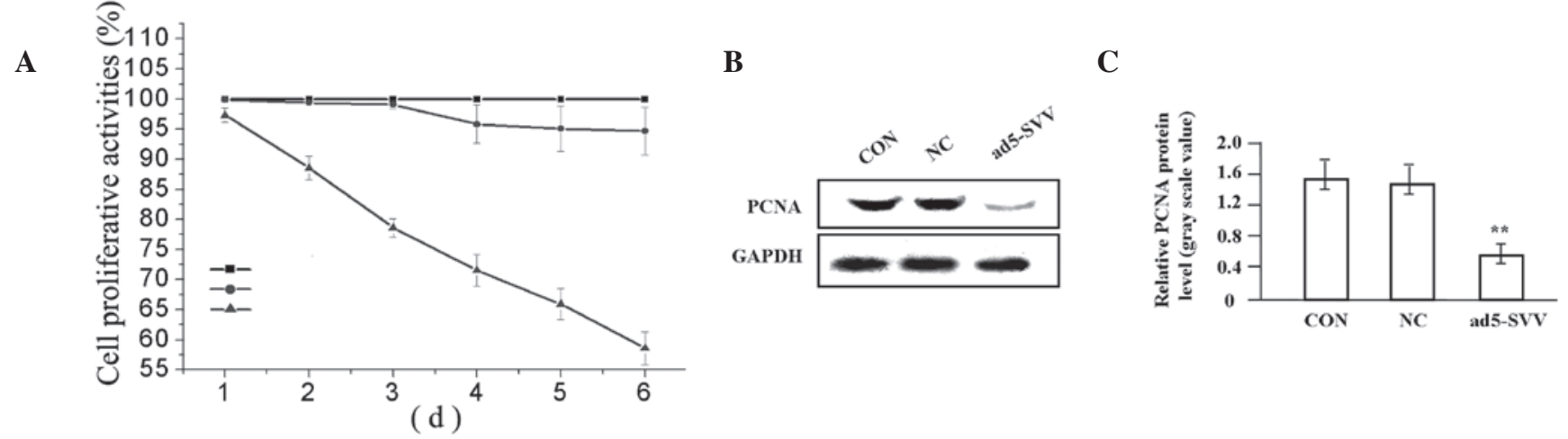

Figure 2. Effect of SVV knockdown on A2780/CP cell proliferation. (A) Effect of SVV knockdown on proliferative activities of A2780/CP cells was examined by MTT, which demonstrated that SVV knockdown significantly inhibited the proliferative activities of A2780/CP cells in a time-dependent manner, compared with those in the NC and CON groups. (B and C) Expression of PCNA protein was examined by western blot analysis, which demonstrated decreased levels of PCNA protein in ad5-SVV compared with NC and CON groups in A2780/CP cells ( $\left.{ }^{* *} \mathrm{P}<0.01\right)$. CON, A2780/CP cells; NC, transfected negative control vectors; Ad5-SVV, transfected Ad5-SVV vectors. SVV, survivin; PCNA, proliferating cell nuclear antigen.

Statistical analysis. Results of each experiment were presented as the mean $\pm \mathrm{SD}$, where applicable. Statistically significant difference in each assay was determined by SPSS version 11.5. Difference in each group was tested for significance using the ANOVA analysis of variance. $\mathrm{P}<0.05$ was considered to indicate a statistically significant difference.

\section{Results}

Effect of SVV knockdown on PCNA, cleaved caspase-3 and MMP-2 expression. To examine whether SVV was knocked down by ad5-SVV and its effect on PCNA, cleaved caspase-3 and MMP-2 mRNA expression in A2780/CP cells, the expression levels of SVV, PCNA, cleaved caspase-3 and MMP-2 mRNA were measured by real-time PCR. As is evident in Fig. 1A, expression levels of SVV, PCNA and MMP-2 mRNA were decreased, while the expression level of cleaved caspase-3 was increased in ad5-SVV compared with the NC and CON groups (each $\mathrm{P}<0.01)$. Western blot analysis of SVV protein expression, revealed a marked downregulation in the ad5-SVV group compared with the NC and CON groups $(\mathrm{P}<0.01$; Fig. 1B and $\mathrm{C})$.

Effect of SVV knockdown on A2780/CP cell proliferation and cisplatin sensitivity. The effect of SVV knockdown on proliferation and cisplatin sensitivity to A2780/CP cells by
MTT was investigated. The results demonstrate that SVV knockdown was identified to significantly inhibit the proliferation of A2780/CP cells in a time-dependent manner, compared with $\mathrm{NC}$ and CON groups (Fig. 2A). PCNA is important for a number of essential cell processes, tumor progression and the outcome of anticancer treatment. To determine whether SVV knockdown suppressed endogenous PCNA expression through translational repression, the expression of PCNA protein was examined by western blot analysis, which revealed a decreased amount of PCNA protein in the ad5-SVV compared with the $\mathrm{NC}$ and $\mathrm{CON}$ groups $(\mathrm{P}<0.01$; Fig. $2 \mathrm{~B}$ and $\mathrm{C})$.

Following this, we pretreated A2780/CP cells in ad5-SVV, $\mathrm{NC}$ and $\mathrm{CON}$ groups with various concentrations of cisplatin $(0.01,0.1,1,10$ and $100 \mu \mathrm{g} / \mathrm{ml})$ and investigated the effect of SVV knockdown on cisplatin sensitivity to A2780/CP cells. Knockdown of SVV markedly enhanced the inhibitory effect and chemotherapeutic sensitivity of cisplatin on A2780/CP cells (each $\mathrm{P}<0.01$ ) (Table I).

Effect of SVV knockdown on A2780/CP cell apoptosis. To determine the effect of SVV knockdown on A2780/CP cell apoptosis, flow cytometric analysis with FITC/PI-Annexin V staining was performed. As demonstrated in Fig. 3A, compared with $\mathrm{NC}$ and $\mathrm{CON}$, the number of apoptotic cells significantly increased and morphological changes, including nuclear condensation, nuclear debris, foam and apoptotic body 
Table I. Effect of SVV knockdown on chemotherapeutic sensitivity of cisplatin to A2780/CP cells indicated by MTT assay (\%)

\begin{tabular}{|c|c|c|c|c|c|}
\hline \multirow[b]{2}{*}{ Group } & \multicolumn{4}{|c|}{ Concentration of cisplatin $(\mu \mathrm{g} / \mathrm{ml})$} & \multirow[b]{2}{*}{100} \\
\hline & 0.01 & 0.1 & 1 & 10 & \\
\hline $\mathrm{CON}$ & $100 \pm 0.00$ & $99.56 \pm 0.88$ & $88.63 \pm 0.75$ & $46.72 \pm 0.43$ & $39.87 \pm 0.56$ \\
\hline $\mathrm{NC}$ & $98.22 \pm 0.15^{\mathrm{a}}$ & $97.83 \pm 0.43^{\mathrm{a}}$ & $86.76 \pm 0.42^{\mathrm{a}}$ & $45.55 \pm 0.56^{\mathrm{a}}$ & $38.34 \pm 0.25^{\mathrm{a}}$ \\
\hline ad5-SVV & $78.56 \pm 0.47^{b}$ & $71.28 \pm 0.52^{\mathrm{b}}$ & $40.05 \pm 0.36^{\mathrm{b}}$ & $32.63 \pm 0.37^{b}$ & $24.57 \pm 0.27^{b}$ \\
\hline
\end{tabular}

${ }^{a} \mathrm{P}>0.05$ vs. $\mathrm{CON}$ group, ${ }^{\mathrm{b}} \mathrm{P}<0.01$ vs. $\mathrm{CON}$ and $\mathrm{NC}$ groups. CON, A2780/CP cells; NC, transfected negative control vectors; Ad5-SVV, transfected Ad5-SVV vectors. SVV, survivin.

A

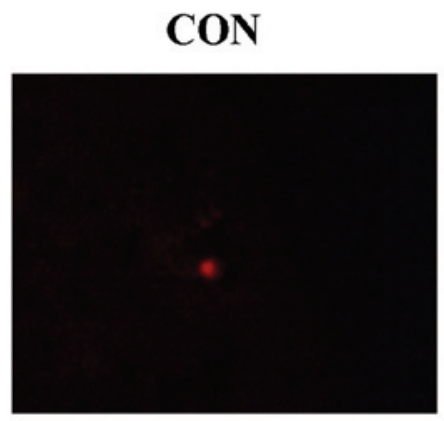

$\mathbf{B}$

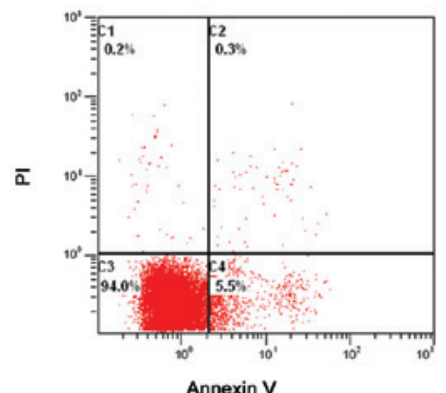

NC
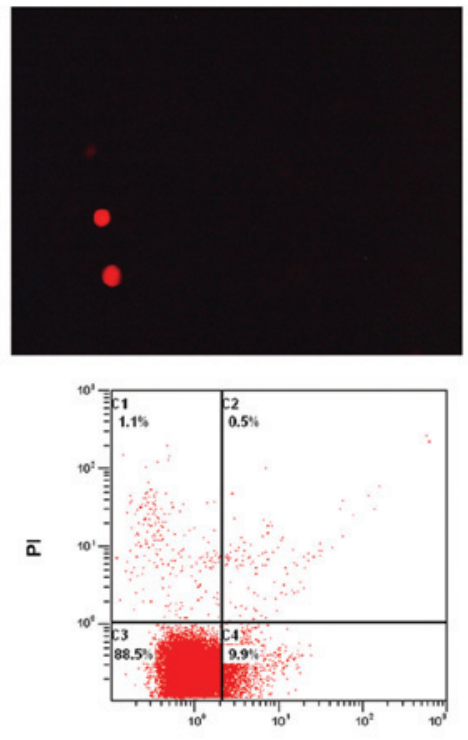

Annexin V
ad5-SVV
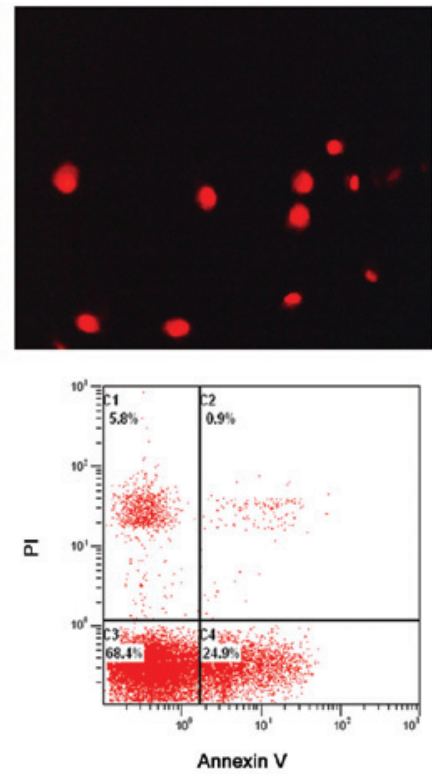

$\mathbf{C}$

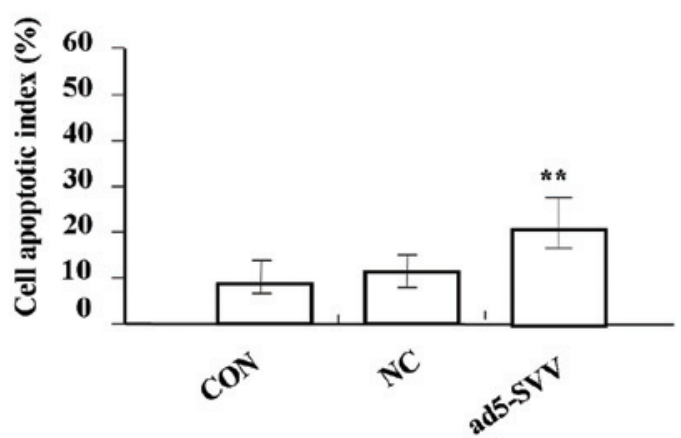

Figure 3. Effect of SVV knockdown on A2780/CP cell apoptosis. (A) To determine the effect of SVV knockdown on A2780/CP cell apoptosis, flow cytometric analysis with PI/FITC-Annexin V staining was performed. Compared with NC and CON, the number of apoptotic cells significantly increased and morphological changes, including nuclear condensation, nuclear debris, foam and apoptotic body formation were observed in the ad5-SVV group. (B and C) Apoptotic index of A2780/CP cells in ad5-SVV was markedly higher than the NC and CON groups ( $\left.{ }^{* *} \mathrm{P}<0.01\right)$. CON, A2780/CP cells; NC, transfected negative control vectors; Ad5-SVV, transfected Ad5-SVV vectors. SVV, survivin.

formation, were identified in the ad5-SVV group. In addition, the apoptotic index of A2780/CP cells in the ad5-SVV group was markedly higher than the $\mathrm{NC}$ and $\mathrm{CON}$ groups $(\mathrm{P}<0.01$; Fig. 3B and C). Western blot analysis was performed to investigate the effect of SVV knockdown on the endogenous expression of pro-caspase- 3 and cleaved caspase- 3 protein. As shown in Fig. 4, pro-caspase-3 and cleaved caspase-3 expression was identified as significantly elevated in the ad5-SVV group, compared with the $\mathrm{NC}$ and $\mathrm{CON}$ groups (each $\mathrm{P}<0.01$ ).

Effect of SVV knockdown on A2780/CP cell invasion. To determine the effect of SVV knockdown on A2780/CP cell 
A

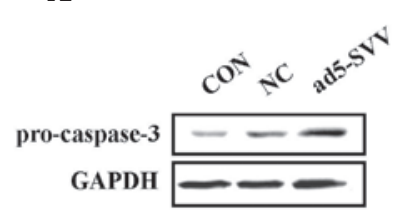

B

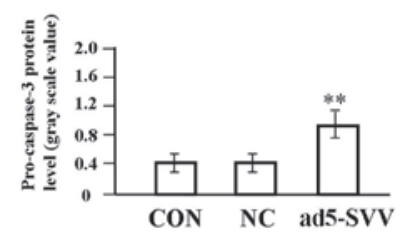

C

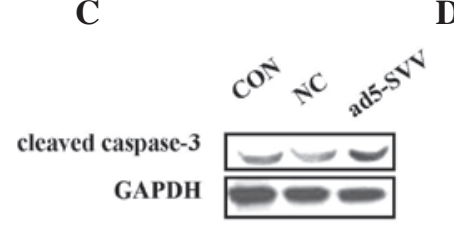

D

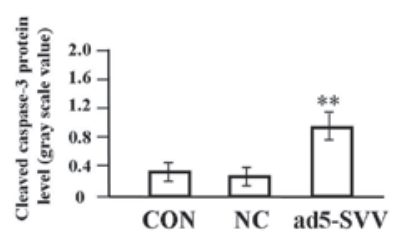

Figure 4. Effect of SVV knockdown on pro-caspase-3 and cleaved caspase-3 protein expression. (A-D) Western blot analysis was performed to investigate the effect of SVV knockdown on the endogenous expression of pro-caspase-3 and cleaved caspase-3 protein and demonstrated that pro-caspase-3 and cleaved caspase-3 expression was significantly elevated in ad5-SVV, compared with the NC and CON groups (each $\left.{ }^{* *} \mathrm{P}<0.01\right)$. CON, A2780/CP cells; NC, transfected negative control vectors; Ad5-SVV, transfected Ad5-SVV vectors. SVV, survivin.

A

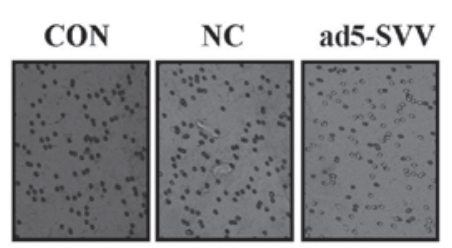

B

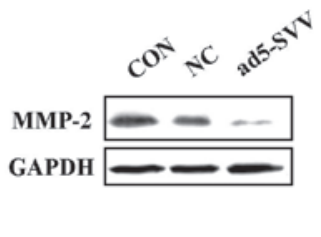

C

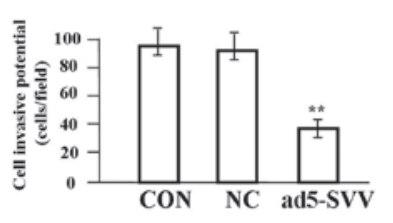

D

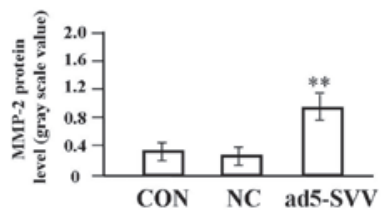

Figure 5. Effect of SVV knockdown on A2780/CP cell invasion. (A) Invasive and metastatic potential in Transwell assay was determined on the basis of the ability of cells to invade a matrix barrier containing laminin and type IV collagen, the major components of the basement membrane. (C) Invasive potential of A2780/CP cells was distinctly decreased in ad5-SVV, compared with the NC and CON groups $\left({ }^{* *} \mathrm{P}<0.01\right)$. (B and D) Western blot analysis was performed to investigate the effect of SVV knockdown on the endogenous expression of MMP-2 protein and revealed that MMP-2 expression was significantly reduced in ad5-SVV, compared with the NC and CON groups $\left({ }^{* *} \mathrm{P}<0.01\right)$. CON, A2780/CP cells; NC, transfected negative control vectors; Ad5-SVV, transfected Ad5-SVV vectors. SVV, survivin; MMP-2 matrix metalloproteinase-2.

invasion, a Transwell assay was performed. Invasive and metastatic potential in the Transwell assay was determined on the basis of the ability of cells to invade a matrix barrier containing laminin and type IV collagen, the major components of the basement membrane. Representative micrographs of Transwell filters are shown in Fig. 5A. The invasive potential of A2780/ $\mathrm{CP}$ cells was markedly decreased in the ad5-SVV group, compared with the $\mathrm{NC}$ and $\mathrm{CON}$ groups $(\mathrm{P}<0.01$; Fig. $5 \mathrm{C})$. Western blot analysis was performed to investigate the effect of SVV knockdown on the endogenous expression of MMP-2 protein. As demonstrated in Fig. 5B and D, MMP-2 expression was identified as significantly reduced in the ad5-SVV group, compared with the $\mathrm{NC}$ and $\mathrm{CON}$ groups $(\mathrm{P}<0.01)$.

\section{Discussion}

The present study demonstrates that shRNA-based knockdown of SVV inhibits proliferation and invasion and induces apoptosis in cisplatin-resistant ovarian cancer cells (A2780/CP). Several studies have demonstrated that the SVV pathway, regulated by other factors, is involved in cell apoptosis in ovarian cancer. Luteinizing hormone (16), FSH (17) and ovarian-specific promoter-2 (18) affect the sensitivity of ovarian cancer cells to chemotherapy and inhibit apoptosis via upregulation of the SVV pathway. Activation of the SVV pathway by interleukin-4 leads to cancer proliferation (19). shRNA targeting against SVV has potential for the treatment of ovarian cancer (20). YM155, a novel small-molecule SVV inhibitor, has demonstrated antitumor activities in human cancer cells (21).

Previous studies have indicated that SVV antisense oligonucleotides induce apoptosis in the drug-resistant ovarian cancer cell line (COC1/DDP) (22). AKT/mTOR/SVV signaling is involved in epithelial ovarian cancer development and cisplatin/paclitaxel-resistant chemotherapy and the downregulation of AKT/SVV may be an effective antitumor therapy (23-25). The present findings have demonstrated that knockdown of SVV enhanced the inhibitory effect and the chemotherapeutic sensitivity of cisplatin to A2780/CP cells and are in agreement with previous reports $(22,23)$, suggesting that SVV is a potential target for therapeutic anticancer drugs. Moreover, accumulating evidence indicates that combinations of targeting SVV with additional compounds, including herbal dietary antioxidants (26), methylseleninic acid (27) and paclitaxel (28), is more effective in the chemoprevention and/ or chemotherapy of ovarian and other types of cancer.

PCNA is a nuclear protein expressed in proliferating cells and may be required for maintaining cell proliferation. Protein levels are used as a marker for the cell proliferation of overall survival (29). MMP-2 is a key enzyme involved in the degradation of type IV collagen and high levels of MMP-2 in tissues is correlated with tumor growth and invasion (30). Caspase-3 is important in the proteolytic cleavage of cell proteins responsible for progression to apoptosis and has the potential to predict the clinical response to chemotherapy (31). The present study has demonstrated a marked decrease in PCNA and MMP-2 expression, but an increase in pro-caspase- 3 and cleaved caspase-3 in group ad5-SVV compared with the NC and CON groups in A2780/CP cells. These observations indicate that the knockdown of SVV may inhibit proliferation and invasion and induce apoptosis in cisplatin-resistant ovarian cancer cells via the downregulation of PCNA and MMP-2 expression and the upregulation of caspase- 3 expression.

To the best of our knowledge, the present study has demonstrated that knockdown of SVV enhances cisplatin-induced 
proliferative activities, induces cell apoptosis and inhibits the invasive potential in A2780/CP cells. Knockdown of SVV contributes to antitumor activity in cisplatin-resistant ovarian cancer cells via the downregulation of PCNA and MMP-2 and upregulation of caspase-3 expression, rendering SVV a potential target for therapeutic anticancer drugs.

\section{References}

1. Eltabbakh G and Awtrey C: Current treatment for ovarian cancer. Expert Opin Pharmacother 2: 109-124, 2001.

2. McKeage M: New-generation platinum drugs in the treatment of cisplatin-resistant cancers. Expert Opin Investig Drugs 14: 1033-1046, 2005.

3. Tewari K, Mehta R, Burger R, et al: Emerging drugs for ovarian cancer. Expert Opin Emerg Drugs 10: 413-442, 2005.

4. Chen H, Hardy TM and Tollefsbol TO: Epigenomics of ovarian cancer and its chemoprevention. Front Genet 2: 67, 2011.

5. Lee S, Choi E, Jin C, et al: Activation of PI3K/Akt pathway by PTEN reduction and PIK3CA mRNA amplification contributes to cisplatin resistance in an ovarian cancer cell line. Gynecol Oncol 97: 26-34, 2005.

6. Ferrandina G, Legge F, Martinelli E, et al: Survivin expression in ovarian cancer and its correlation with clinico-pathological, surgical and apoptosis-related parameters. Br J Cancer 92: 271-277, 2005.

7. Liguang Z, Peishu L, Hongluan M, et al: Survivin expression in ovarian cancer. Exp Oncol 29: 121-125, 2007.

8. No JH, Jeon YT, Kim YB, et al: Quantitative detection of serum survivin and its relationship with prognostic factors in ovarian cancer. Gynecol Obstet Invest 71: 136-140, 2011.

9. Qian X, Xi X and Li L: Nuclear survivin is associated with malignant potential in epithelial ovarian carcinoma. Appl Immunohistochem Mol Morphol 19: 126-132, 2011.

10. Kleinberg L, Flørenes VA, Silins I, et al: Nuclear expression of survivin is associated with improved survival in metastatic ovarian carcinoma. Cancer 109: 228-238, 2007.

11. Zaffaroni N, Pennati M, Colella G, et al: Expression of the antiapoptotic gene survivin correlates with taxol resistance in human ovarian cancer. Cell Mol Life Sci 59: 1406-1412, 2002.

12. Felisiak-Golabek A, Rembiszewska A, Rzepecka IK, et al: Nuclear survivin expression is a positive prognostic factor in taxane-platinum-treated ovarian cancer patients. J Ovarian Res 4: 20,2011

13. Ma X, Wang S, Zhou J, et al: Induction of apoptosis in human ovarian epithelial cancer cells by antisurvivin oligonucleotides. Oncol Rep 14: 275-279, 2005.

14. Caldas H, Jaynes FO, Boyer MW, et al: Survivin and Granzyme $\mathrm{B}$-induced apoptosis, a novel anticancer therapy. Mol Cancer Ther 5: 693-703, 2006.

15. Zhang B, Pan JS, Liu JY, et al: Effects of chemotherapy and/or radiotherapy on survivin expression in ovarian cancer. Methods Find Exp Clin Pharmacol 28: 619-625, 2006.
16. Zhang Z, Liao $\mathrm{H}$, Chen $\mathrm{X}$, et al: Luteinizing hormone upregulates survivin and inhibits apoptosis in ovarian epithelial tumors. Eur J Obstet Gynecol Reprod Biol 155: 69-74, 2011.

17. Huang Y, Jin H, Liu Y, et al: FSH inhibits ovarian cancer cell apoptosis by up-regulating survivin and down-regulating PDCD6 and DR5. Endocr Relat Cancer 18: 13-26, 2010.

18. Tu CH, Liu WP, Dong M, et al: Protection of CHO cells by transfer of survivin driven by ovarian-specific promoter OSP-2. Mol Biol Rep 38: 2323-2328, 2011.

19. Roca H, Craig MJ, Ying C, et al: IL-4 induces proliferation in prostate cancer PC3 cells under nutrient-depletion stress through the activation of the JNK-pathway and survivin up-regulation. J Cell Biochem 113: 1569-1580, 2012.

20. Xing J, Jia CR, Wang Y, et al: Effect of shRNA targeting survivin on ovarian cancer. J Cancer Res Clin Oncol 138: 1221-1229, 2012.

21. Nakahara T, Kita A, Yamanaka K, et al: Broad spectrum and potent antitumor activities of YM155, a novel small-molecule survivin suppressant, in a wide variety of human cancer cell lines and xenograft models. Cancer Sci 102: 614-621, 2011.

22. Zheng F, Ruan F, Xie XK, et al: Apoptosis of drug-resistant human ovarian carcinoma cell line COC1/DDP induced by survivin antisense oligonucleotides. Chin Med J (Engl) 119: 1572-1575, 2006

23. Zhang HY, Zhang PN and Sun H: Aberration of the PI3K/AKT/ mTOR signaling in epithelial ovarian cancer and its implication in cisplatin-based chemotherapy. Eur J Obstet Gynecol Reprod Biol 146: 81-86, 2009.

24. Weng D, Song X, Xing H, et al: Implication of the Akt2/survivin pathway as a critical target in paclitaxel treatment in human ovarian cancer cells. Cancer Lett 273: 257-265, 2009.

25. Xing H, Weng D, Chen G, et al: Activation of fibronectin/ PI-3K/Akt2 leads to chemoresistance to docetaxel by regulating survivin protein expression in ovarian and breast cancer cells. Cancer Lett 261: 108-119, 2008.

26. Raj MH, Abd Elmageed ZY, Zhou J, et al: Synergistic action of dietary phyto-antioxidants on survival and proliferation of ovarian cancer cells. Gynecol Oncol 110: 432-438, 2008.

27. Azrak RG, Frank CL, Ghadersohi A, et al: Silencing survivin results in synergy between methylseleninic acid and paclitaxel against skov3 ovarian cancer cells. Cancer Biol Ther 7: 1901-1908, 2008.

28. Vivas-Mejia PE, Rodriguez-Aguayo C, Han HD, et al: Silencing survivin splice variant $2 \mathrm{~B}$ leads to antitumor activity in taxane - resistant ovarian cancer. Clin Cancer Res 17: 3716-3726, 2011.

29. Reitmaier M, Rudlowski C, Biesterfeld S, et al: Comparative studies on the biological significance of the marker for proliferation Ki-67-antigen and PCNA in primary ovarian carcinoma. Zentralbl Gynakol 122: 361-367, 2000 (In German).

30. Brinckerhoff CE, Rutter JL and Benbow U: Interstitial collagenases as markers of tumor progression. Clin Cancer Res 6: 4823-4830, 2000.

31. Flick MB, O'Malley D, Rutherford T, et al: Apoptosis-based evaluation of chemosensitivity in ovarian cancer patients. J Soc Gynecol Investig 11: 252-259, 2004. 